Frawley, J, Davidson, S, Anheyer, D and Jackson, D

Prevalence and characteristics of Complementary and Alternative Medicine use by Australian children

Frawley, J, Davidson, S, Anheyer, D and Jackson, D () Prevalence and characteristics of Complementary and Alternative Medicine use by Australian children. Journal of Paediatrics and Child Health

This version is available: https://radar.brookes.ac.uk/radar/items/0df1de96-4e70-4a4d-9a92-574fe5f936cf/1/

Deposited on RADAR: March 2017

Copyright (C) and Moral Rights are retained by the author(s) and/ or other copyright owners. A copy can be downloaded for personal non-commercial research or study, without prior permission or charge. This item cannot be reproduced or quoted extensively from without first obtaining permission in writing from the copyright holder(s). The content must not be changed in any way or sold commercially in any format or medium without the formal permission of the copyright holders.

This document is the postprint version of the journal article. Some differences between the published version and this version may remain and you are advised to consult the published version if you wish to cite from it. 


\title{
Prevalence and characteristics of complementary and alternative medicine use by Australian children
} Authors

Jane Frawley, Sandy Davidson, Dennis Anheyer, Debra Jackson

\begin{abstract}
This study was conducted to evaluate the prevalence and characteristics of complementary and alternative medicine (CAM) use, among Australian children and adolescents within the previous 12 months.

Methods: Parents with children up to the age of 18 years were recruited from online parenting groups. Questions addressed demographic factors, socioeconomic status, conventional health service use, including vaccination status, and use of CAM.
\end{abstract}

Results: A total of 149 parents responded to the study of which $73.8 \%(n=110)$ children had visited a CAM practitioner or used a CAM product in the previous 12 months. The two most frequently visited CAM practitioners were naturopath/herbalist (30.4\%) and chiropractor (18.4\%). The most commonly used products were vitamins/minerals (61.7\%), and herbal medicine (38.8\%). Children had also consulted with a general practitioner (89.8\%), community health nurse (31.29\%) and paediatrician (30.3\%) over the same period. A total of $52 \%$ of parents did not disclose their child's use of CAM to their medical provider. Children's vaccination status was less likely to be up-to-date if they visited a CAM practitioner (OR 0.16; CI 0.07, 0.36; $\mathrm{p}<0.001$ ) or used a CAM product (OR 0.25; CI 0.09, 0.64; $\mathrm{p}=0.004$ ).

Conclusion: Despite a lack of high quality research for efficacy and safety, many children are using CAM products and practices in parallel with conventional health services, often without disclosure. This highlights the need to initiate conversations with parents about their child's use of CAM in order to ensure safe, coordinated patient care. The association between vaccine uptake and CAM use requires further investigation. 
Keywords: child health, complementary and alternative medicine, immunisation, health services research

\section{Introduction}

Despite a lack of rigorous evidence to determine efficacy, safety and sufficient dosing regimes ${ }^{1,2}$, children's use of complementary and alternative medicine (CAM) - a range of products and practices generally considered to be outside the dominant medical paradigm ${ }^{3}$ - has increased in popularity in recent years. ${ }^{4} \mathrm{~A}$ recent systematic review of 58 studies from 19 countries found the prevalence of CAM use for children varied widely from $10.9 \%$ to $87.6 \%$ for lifetime use and from $8 \%$ to $48.5 \%$ for current use, depending on nationality and CAM modalities included. ${ }^{4}$ The majority of studies focused on CAM use in general (i.e. all modalities combined) and very few investigated individual CAM practitioners (i.e. naturopaths, chiropractors, traditional Chinese practitioners), or individual CAM treatments such as herbal medicine, vitamin or mineral use. ${ }^{4}$

The two most recent Australian studies to explore overall CAM use for children (overall, not for one specific condition such as cancer) occurred over a decade ago. Smith and Eckert (2006) found 18\% of children had used a CAM product or consulted a CAM practitioner in the previous 12-months, most commonly to prevent illness (39\%), and to treat musculoskeletal (22\%), respiratory $(20 \%)$ and skin conditions (18\%). ${ }^{5}$ The second study compared CAM use amongst children at hospital outpatient clinics in Australia and Wales (UK), and found $51 \%$ of the Australian children had used CAM in the previous 12 months, with $63 \%$ of parents not disclosing this use to the child's primary care practitioner. ${ }^{6}$

The use of CAM in children and adolescents constitutes specific legal, ethical and clinical challenges due to the lack of high quality evidence for the safety and efficacy of CAM in these populations ${ }^{7}$, and largely unregulated CAM professions. ${ }^{8,9}$ Whilst recent research in Europe and the United States (US) has highlighted some of the characteristics of children's CAM use, very little is currently known about the prevalence, drivers and features of CAM use by Australian children. Additionally, there is a dearth of research investigating the 
interface between visits to conventional medical practitioners, such as general practitioners (GPs) and paediatricians; and conventional public health services, such as childhood immunisation and CAM use. Accordingly, the aim of this study was to evaluate health service utilisation, including use of CAM, among Australian children and adolescents within the previous 12 months.

\section{Materials and methods}

\section{Sample}

The study sample was obtained via an online survey of Australian parents. The survey was posted twice over a period of four weeks on a national parenting website (BubHub) as well as Facebook parenting groups (posted once in four different groups), and parents of children up to the age of 18 years were invited to participate. The 32-item questionnaire included both closed and open-ended questions, addressing areas of demographics, conventional health service use and CAM practitioner and product use, and was estimated to take 10-15 minutes to complete. Ethics approval was gained from the relevant ethics committees at the XXXXXXXX (Australia) and XXXXXX (Australia).

\section{Demographic measures}

Parents were asked about their gender, marital status, age, income, employment and highest level of educational qualification completed. Participants were also asked to classify their place of residence as either urban (capital city or major metropolitan center with a population $>100,000$ ) or non-urban (population $<100,000)$ and if they had private health insurance or a government health care card.

\section{Health service use}

Parents were asked about the health services they had visited for their children's health needs in the previous 12 months. Specifically, parents were asked if they took their child/children to visit a GP, paediatrician, community health nurse or other medical specialist in the past 12 months and additionally, if they took their child/children to visit a CAM practitioner in the previous 12 months (including a naturopath/herbalist, nutritionist, osteopath, chiropractor, massage therapist, 
traditional Chinese medicine practitioner and homeopath). Parents were also asked if they had used a CAM product for their child/children in the previous 12 months, including herbal medicines, vitamins and minerals, aromatherapy oils or homoeopathic medicine. Parents were also asked if their children were vaccinated. Parents were asked which information sources they trusted when making decisions about the use of CAM and if they disclosed their child's use of CAM to their child's primary health practitioner.

\section{Health conditions}

Parents who indicated that they had visited a CAM practitioner or used a CAM product for their child in the previous 12 months were given a range of common childhood health conditions and asked to indicate what the CAM was used for. These conditions included general wellbeing, teething, colic, cradle cap, teething, sleeping difficulties, nappy rash, diarrhoea, constipation, stomach discomfort, anxiety, eczema, asthma, food intolerance, ear infections, growing pains, vaccine preventable disease (i.e. chicken pox, whooping cough), other infectious disease (i.e. impetigo, glandular fever, hand foot and mouth), autoimmune disease, acne, period pain or to supplement the diet of a fussy eater.

\section{Statistical analysis}

The characteristics of parents choosing to visit a CAM practitioner and/or to use a CAM product for their child during the last 12 months were investigated, and relationships were determined using a chi-square analysis. Identification of significant covariates was also determined through univariate logistic regression between all possible predictors (i.e. the demographic, health care variables and information sources) and CAM practitioner and/or product use. All the demographic and health service utilisation variables listed above were entered into a model and then a stepwise backward elimination process was employed, using a likelihood ratio test, to eventually produce the most parsimonious model. Statistical significance was set at $\mathrm{p}<0.05$. All analyses were conducted using statistical program STATA 14.1 (StataCorp LP, College Station, TX, USA).

\section{Results}


A total of 149 parents responded to the online study of which $97.3 \%$ were female (as shown in Table 1). Parents were more likely to have one $(n=62,41.3 \%)$ or two $(n=73,48.7 \%)$ children. Just over half of the respondents had a child aged between two and five years $(n=76,51.0 \%)$, with $37 \%$ having a child aged between $6-12,36 \%$ had a child aged up to two years and $10 \%$ had a child aged 12-18 years. Respondents were more commonly aged between 35 and 44 years $(\mathrm{n}=81,54.4 \%)$, married or living with a partner $(\mathrm{n}=135,90.6 \%)$, were financially comfortable ( $n=84,56.8 \%)$, had private health insurance for both hospital and extras $(n=78,54.9 \%)$, did not have a health care card $(n=116,78.9 \%)$ and had attained a degree or postgraduate degree as their highest education qualification $(\mathrm{n}=117,78.5 \%)$ (see Table 1). A total of 99 parents $(66.4 \%)$ indicated that their children's vaccination status was up to date.

Overall, $110(73.8 \%)$ of parents had taken their child/children to visit a CAM practitioner or given their child/children a CAM product in the previous 12 months. Of this, $72(48.3 \%)$ parents had taken their child/children to consult a CAM practitioner and 102 (68.5\%) of parents had given their child a CAM product. Individual CAM practitioners consulted included naturopath/herbalist $(n=45,30.4 \%)$, chiropractor $(n=27,18.4 \%)$, osteopath $(n=22,15.0 \%)$, homeopath $(\mathrm{n}=17,11.6 \%)$, traditional Chinese practitioner $(\mathrm{n}=12,8.2 \%)$, nutritionist $(n=10,6.8 \%)$ and massage therapist $(n=6,4.1 \%)$. CAM products included vitamins and minerals $(n=92,61.7 \%)$, herbal medicine $(n=57,38.8 \%)$, essential oils $(n=44,29.9 \%)$ and homoeopathic medicines $(n=17,11.6 \%)$. Excluding vitamins and minerals, $51.6 \% \quad(\mathrm{n}=77)$ of parents gave their child/children herbal medicine, homeopathic medicine and/or used essential oils.

Parents had also consulted a GP $(n=132,89.8 \%)$, community health nurse $(n=46$, $31.29 \%)$, paediatrician $(n=44,30.3 \%)$, and other medical specialist $(n=57$, $38.8 \%)$ in the previous 12 months. Only $5.4 \%(n=8)$ of parents had taken a child to visit a CAM practitioner, but not a conventional health practitioner, in the previous 12 months. A total of $52 \%$ of parents did not disclose their child's use of CAM to their medical provider with the most common reasons being 'the doctor 
did not ask' (50.0\%), 'I am concerned the doctor isn't knowledgeable about complementary medicine' (40.5\%), 'I didn't think it was important' (29.7\%), 'I was concerned about receiving a negative response' (28.4\%), and 'I have not visited the doctor since commencing complementary medicine treatment' $(28.38 \%)$.

When looking for information about the use of CAM for children, parents trusted certain information sources, including friends and family (61.4\%), CAM practitioners (55.6\%), GPs (39.2\%), online parenting website or groups (37.4\%), health food store attendants (23.0\%), nurse/midwives (17.6\%), general Internet (17.1\%), paediatricians (16.8\%), newspaper (5.7\%) and radio/television (2.4\%). Overall though, $84.5 \%$ of parents said that they were influenced by their personal experiences of using CAM.

Table 2 details the conditions/reasons that CAM was used. The top 10 reasons reported were general wellbeing $(n=73,66.4 \%)$, colds/flu $(n=59,53.6 \%)$, supplement diet of a fussy eater $(n=27,24.5 \%)$, teething $(n=25,22.7 \%)$, sleeping $(n=18,16.4 \%)$, stomach discomfort $(n=17,15.5 \%)$, constipation $(n=15,13.6 \%)$, anxiety $(n=13,11.8 \%)$, diarrhoea $(n=13,11.8 \%)$, and eczema $(n=13,11.8 \%)$.

Chi square analysis found children's use of CAM products was associated with parental age, area of residence, marital status, and education $(\mathrm{p}<0.05)$. Both the use of CAM products and visits to CAM practitioners were associated with childhood vaccination status $(\mathrm{p}<0.05)$. Multiple logistic regression found that children's vaccination status was less likely to be up to date if they visited a CAM practitioner (OR 0.16; CI 0.07, 0.36; p<0.001) (Table 3) or used CAM products (OR 0.25; CI 0.09, 0.64; p=0.004) (Table 4), however no other demographic or health service variables were significant.

\section{Discussion}

Our study found a very high use of CAM amongst children aged <18years with over two thirds of parents using CAM for their children in the previous 12 
months. Of this, $48.3 \%$ had consulted a CAM practitioner and $68.5 \%$ had used a CAM product. This finding is higher than the prevalence of one year CAM use previously reported in Australia $(18 \%-51 \%)$ a decade ago5,6, however it is difficult to compare our results with these two large population based studies and further research is warranted. On the surface, worldwide paediatric CAM use tends to vary significantly from country to country and arguably, region to region. ${ }^{10,11,12}$ Whilst large US studies have demonstrated the growing use of CAM amongst children in recent years ${ }^{1}$, rates of use appear to be lower than those found in Europe, and those reported by this study. In 2007 for example, the National Health Interview Survey (NHIS) showed that about 12\% of children had used some kind of CAM including osteopathy, herbal medicine or homeopathy, within the last 12 months. ${ }^{11}$ However, it should be noted that this NHIS analysis does not include vitamins and minerals that were reported separately by Dwer et al (2013) to be utilised by $37 \%$ of children and these combination of these rates would provide a rate of use more in line with European studies and the results presented here. ${ }^{12}$

Pluralistic health service use was evident among children in our study. Just under half of all children who had visited a GP in the previous 12 months had also consulted a CAM practitioner and $68 \%$ had used a CAM product in the same time period. Similarly, half of all children who had visited a paediatrician had also consulted a CAM practitioner and over two-thirds had used a CAM product in the last 12 months. This corresponding but often parallel health service use is significant and the consultation may offer an opportunity to enquire about CAM use. This is especially appropriate given that over half of all parents did not disclose their children's use of CAM, with the main reason being that the doctor did not ask. Whilst other reasons for not discussing CAM use exist, for example fear of being admonished, this provides important insights for primary health care practitioners, and it is important to routinely inquire about CAM use and initiate an open and non-judgmental conversation about this use in order to build patient/practitioner trust and respect. 
CAM was used for a wide variety of health complaints and to augment general health, despite a lack of high quality evidence for efficacy and safety in children. ${ }^{7}$ Research illustrates that parents may favour CAM for their children's minor health complaints, as they perceive CAM to be 'natural', equating this with being safe. ${ }^{13}$ Our study found that parents also frequently utilise their friend and family networks for advice and information regarding CAM use for their children ${ }^{13}$ and as a result may not be accessing reliable and accurate information. Follow-up research is needed to determine which individual CAMs are being used by children in order to establish if safety concerns exist. Further to this, very little research has been conducted on CAM in a paediatric population, leaving practitioners and parents to make treatment decisions despite a certain level of ambiguity. ${ }^{7}$ Despite the complex legal and ethical considerations, more research is warranted on the safety and efficacy of CAM for children as many parents have already embraced the use of common CAM practices and products for their children.

The results reported here demonstrate that both CAM practitioner and CAM product use by children in the previous 12 months are associated with lower uptake of childhood vaccination. This finding is in line with a recent review that found a relationship between lower rates of vaccination and visits to CAM practitioners and the use of CAM products. ${ }^{14}$ It is difficult to determine the explicit reasons for this association and more research is needed to establish if it is a result of confounding, due to common factors associated with CAM use (education level, income, distrust of the medical system), or a direct and independent relationship. Wardle et al (2016) also reviewed CAM practitioner attitudes to vaccination and found that whilst objection was significantly higher amongst CAM practitioners than mainstream medical practitioners, there was no default position and many CAM practitioners held positive attitudes towards vaccination. Vaccine-hesitant parents were also found to be more likely to trust vaccine information from CAM practitioners as opposed to mainstream medical practitioners. ${ }^{14}$ This may present an opportunity for CAM practitioners to have open, rational, evidence-based conversations with vaccine-hesitant parents about the benefits and risks of vaccination. 


\section{Limitations}

The increasing popularity of CAM may be partially responsible for the high prevalence of CAM use reported in this study; however, it is unlikely that it is solely responsible for this finding. It is possible that parents who used CAM for their children were more inclined to complete the online survey, which may have artificially increased the prevalence of CAM use. Further, respondents were more likely to be financially comfortable, have attained a tertiary education and have private health insurance cover, which makes it impossible to extrapolate these results to the wider community. The experiences and practices of parents from other socio-demographic backgrounds may be different and future research should capture a wider range of economic backgrounds. Parents were asked about health service use for their child/children and not for each individual child, which only gives an indication of the use of health services for children in the household generally and not for each child (if more than one). This may have altered prevalence rates. Lastly, the lower rates of vaccination found in this study may also have been affected by responder bias. Whilst our research is not representative of all Australian children, it is the first paper in over 10 years to detail the characteristics of CAM use by children in the general Australian population and therefore reports important findings. Larger, nationally representative studies are needed to further investigate these findings.

\section{Conclusion}

Many Australian children are visiting CAM practitioners and/or using CAM products despite a lack of high-quality evidence for the safety and efficacy of these services and products. Further, many parents are reluctant to disclose CAM use with their child's primary health care provider, creating barriers for the provision of coordinated, safe health care. Further research is required to determine the key drivers for CAM use amongst children and to deepen our understanding of the association with vaccine status.

Acknowledgements: 
This research was supported by a seed grant from XXXXXXX. The authors would like to thank all parents who participated in this study. All authors participated in the preparation of this manuscript.

\section{References}

1. Kemper KJ, Vohra S, Walls R, et al. American Academy of Pediatrics. The use of complementary and alternative medicine in pediatrics. Pediatrics 2008; 122: 1374-86.

2. Meyer S, Gortner L, Larsen A, et al. Complementary and alternative medicine in paediatrics: a systematic overview/synthesis of Cochrane Collaboration reviews. Swiss Medical Weekly 2013; 143: w13794.

3. Adams J, Andrews G, Barnes J, Broom A, Magin P, eds. Traditional, Complementary and Integrative Medicine: An International Reader. Basingstoke, Palgrave MacMillan, 2012.

4. Italia S, Wolfenstetter SB, Teuner CM. Patterns of complementary and alternative medicine (CAM) use in children: a systematic review. European Journal of Pediatrics 2014; 173: 1413-28.

5. Smith C, Eckert K. Prevalence of complementary and alternative medicine and use among children in South Australia. Journal of Paediatrics and Child Health 2006; 42: 538-543.

6. Cincotta DR, Crawford NW, Lim A, et al. Comparison of complementary and alternative medicine use: reasons and motivations between two tertiary children's hospitals. Archives of Disease in Childhood 2006; 91: 153-8.

7. Gilmour J, Harrison C, Cohen MH, Vohra S. Pediatric use of complementary and alternative medicine: legal, ethical, and clinical issues in decisionmaking. Pediatrics 2011; 128 Suppl 4: S149-54.

8. Jarvis A, Perry R, Smith D, Terry R, Peters S. General practitioners' beliefs about the clinical utility of complementary and alternative medicine. Primary Health Care Research \& Development 2015; 16: 246-53. 
9. Wardle J, Steel A, Adams J. A review of tensions and risks in naturopathic education and training in Australia: a need for regulation. Journal of Alternative and Complementary Medicine 2012; 18: 363-70.

10. Zuzak TJ, Bonkova J, Careddu D, et al. Use of complementary and alternative medicine by children in Europe: published data and expert perspectives. Complementary Therapies in Medicine 2013; 21 Suppl 1: S34-47.

11. Barnes PM, Bloom B, Nahin RL. Complementary and alternative medicine use among adults and children: United States, 2007. National Health Statistics Reports 2008: 1-23.

12. Dwyer J, Nahin RL, Rogers GT, et al. Prevalence and predictors of children's dietary supplement use: the 2007 National Health Interview Survey. The American Journal of Clinical Nutrition 2013; 97: 1331-37.

13. Pike A, Etchegary H, Godwin M, et al. Use of natural health products in children: qualitative analysis of parents' experiences. Canadian Family Physician Medecin de Famille Canadien 2013; 59: e372-8.

14. Wardle J, Frawley J, Steel A, Sullivan E. Complementary medicine and childhood immunisation: A critical review. Vaccine 2016; 34: 4484-500. 